\title{
Blocking TNF $\alpha$ attenuates progressive cartilage matrix degradation in inflammatory arthritis
}

\author{
JINSUNG PARK ${ }^{1,2^{*}}$, HYOSUN PARK $^{1,2^{*}}$, YOUNG LIM LEE $^{1}$, SUBIN WEON $^{1,2}$, \\ YONG-GIL KIM ${ }^{3}$, JAE-HYUK YANG ${ }^{4}$, BORA NAM ${ }^{1,5}$, SUNGSIN JO $^{1}$ and TAE-HWAN KIM ${ }^{1,2,5}$
}

\author{
${ }^{1}$ Hanyang University Institute for Rheumatology Research; ${ }^{2}$ Department of Translational Medicine, Graduate School \\ of Biomedical Science and Engineering, Hanyang University, Seoul 04763; ${ }^{3}$ Division of Rheumatology, \\ Department of Medicine, University of Ulsan College of Medicine, Asan Medical Center, Seoul 05505; \\ ${ }^{4}$ Department of Orthopedics, Hanyang University Guri Hospital, Guri, Gyeonggi 11923; ${ }^{5}$ Department of \\ Rheumatology, Hanyang University Hospital for Rheumatic Diseases, Seoul 04763, Republic of Korea
}

Received June 30, 2020; Accepted April 27, 2021

DOI: 10.3892/etm.2021.10240

\begin{abstract}
Because damage to hyaline cartilage is irreversible, relieving progressive cartilage destruction is an important therapeutic approach for inflammatory arthritis. In the present study, human hyaline chondrocytes were isolated from total knee replacements of 15 patients with osteoarthritis (OA) and three with rheumatoid arthritis (RA). Synovial fluid of OA $(n=25)$ and RA $(n=34)$ were collected to measure tumor necrosis factor $\alpha(\mathrm{TNF} \alpha)$ using ELISA. Consistent with previous studies, the synovial fluid exhibited high TNF $\alpha$ levels and hyaline cartilage was severely destroyed in patients with RA. TNF $\alpha$-treated chondrocytes were used as model for inflammatory arthritis. TNF $\alpha$ did not influence proliferation or extracellular matrix expression in chondrocytes, but induced matrix metalloproteinase (MMP)1, 3 and 13 expression levels in chondrocytes, which was accompanied by activation of nuclear factor- $\kappa \mathrm{B}$ signaling. During chondrogenic
\end{abstract}

Correspondence to: Professor Tae-Hwan Kim, Department of Rheumatology, Hanyang University Hospital for Rheumatic Diseases, 222-1 Wangsimni, Seongdong, Seoul 04763, Republic of Korea E-mail: thkim@hanyang.ac.kr

Dr Sungsin Jo, Hanyang University Institute for Rheumatology Research, 222-1 Wangsimni, Seongdong, Seoul 04763, Republic of Korea

E-mail: joejo0517@gmail.com

${ }^{*}$ Contributed equally

Abbreviations: OA, osteoarthritis; RA, rheumatoid arthritis; $\mathrm{TNF} \alpha$, tumor necrosis factor $\alpha$; MMP, matrix metalloproteinase; NF- $\kappa$, nuclear factor $\kappa \mathrm{B}$; SOX9, SRY-box transcription factor 9; COL2, collagen type 2; ACAN, aggrecan

Key words: inflammatory arthritis, chondrocytes, chondrogenic differentiation, metabolic shift, $\mathrm{TNF} \alpha$, anti-TNF $\alpha$ inhibitor differentiation, TNF $\alpha$ attenuated mRNA expression levels of anabolic factors (collagen type 2 and aggrecan) and enhanced mRNA expression of catabolic factors (MMP1, MMP3 and $M M P 13)$ in chondrocytes. Moreover, anti-TNF $\alpha$ agents (Golimumab) inhibited the TNF $\alpha$-induced metabolic shift in chondrocytes and chondrogenic differentiation. The present study revealed a mechanism by which $\mathrm{TNF} \alpha$ may induce metabolic shift in chondrocytes, leading to progressive chondrocyte destruction.

\section{Introduction}

Hyaline cartilage can be damaged by trauma and is degraded in different forms of arthritis $(1,2)$. Once damaged, cartilage has a limited capacity to repair and does not fully regenerate $(3,4)$. In past decades, efforts have been made to achieve functional repair of hyaline cartilage and to regenerate hyaline cartilage-like tissue, but these clinical attempts have consistently failed $(5,6)$. This may be due to a lack of knowledge regarding the formation and maintenance of distinct features of hyaline cartilage and the components of its extracellular matrix. Therefore, a biological understanding of chondrocytes derived from human joints and extensive research may be necessary to achieve these goals.

Cartilage is an unusual tissue in that chondrocytes can be anabolic (synthesize matrix) or catabolic (degrade matrix) (7). In adult cartilage, a balance exists between the synthesis and degradation of the cartilaginous extracellular matrix. However, inflammation can cause an imbalance between expression of anabolic [aggrecan (ACAN) and collagen type II (COL2)] and catabolic [matrix metalloproteinases (MMPs)] factors in chondrocyte tissue (8-10). A severe imbalance between anabolic and catabolic chondrocyte factors leads to progressive degradation of the cartilage matrix (11-13). Specifically, pro-inflammatory stimuli in chondrocytes drive poor regenerative capacity by inducing catabolic molecules (14).

Cartilage destruction is a key characteristic of degenerative joint diseases, particularly osteoarthritis (OA); it also features in chronic inflammatory joint diseases, such as rheumatoid 
arthritis (RA) (2). Tumor necrosis factor $\alpha$ (TNF $\alpha$ ) is a pleotropic cytokine that plays key regulatory roles in inflammation and the response to cellular stressors such as oxidative stress $(15,16)$. It also serves a vital role in the pathological process of inflammatory changes by promoting release of MMP and downregulation of SRY-box transcription factor 9 (SOX9), COL2 and ACAN expression, eventually leading to extracellular matrix destruction in hyaline cartilage (17). Therefore, TNF $\alpha$ has been highlighted as a pathological factor in both OA and RA. Previous studies have reported that TNFa serves an important role in hyaline cartilage destruction in both OA and RA pathophysiology $(18,19)$. Therefore, TNF $\alpha$ has proven to be a potential effective therapeutic target for patients with RA (15).

The present study defined specific surface markers of primary chondrocytes derived from human OA hyaline cartilage and investigated the role of TNF $\alpha$ in the chondrogenesis of hyaline cartilage.

\section{Materials and methods}

Human $O A$ and RA subjects. The present study was performed in accordance with Hanyang University Hospital Institutional Review Board guidelines and approved by the Ethics Committee of Hanyang University Hospital (approval no. 2017-05-003) and Hanyang University Guri Hospital (approval no. 2018-07-024). Written informed consent was obtained from all subjects.

Between April 2018 and May 2019, 15 patients with OA (9 females and 6 males; mean age, $70.5 \pm 8.7$ years) and three with RA (all females; mean age, $57.1 \pm 11.3$ years) were enrolled and surgical samples were obtained from total knee replacement at Hanyang University Guri Hospital. Both OA and RA surgical knee samples were fixed at room temperature (RT) for two weeks with $10 \%$ formalin, decalcified with $10 \%$ formic acid and embedded routinely in a paraffin block. The paraffin block was sectioned at a thickness of $3.5 \mu \mathrm{m}$ and stained by hematoxylin and eosin, Safranin O and Toluidine blue. The section slides were deparaffinized in the tissue in $100 \%$ neoclear (cat. no. 109843; Merck) for $10 \mathrm{~min}$ and rehydration in serial ethanol dilution $(100,90,80,70$, and $50 \%$ ethanol) for $2 \mathrm{~min}$ each step, and washed with tap water for $5 \mathrm{~min}$. For $\mathrm{H} \& \mathrm{E}$ staining, the slides were stained with $100 \%$ hematoxylin for $3 \mathrm{~min}$, washed with tap water for $10 \mathrm{~min}, 100 \%$ eosin stained for $3 \mathrm{~min}$, washed with tap water, and mounted with a permanent mounting medium (cat. no. H-5000; Vector Lab). For Safranin O staining, the slides were stained with $0.1 \%$ fast green stained for $5 \mathrm{~min}$, washed with $1 \%$ acetic acid one tapping, washed with tap water for $1 \mathrm{~min}, 0.1 \%$ Safranin $\mathrm{O}$ for $6 \mathrm{~min}$, washed with tap water for $1 \mathrm{~min}$, and mounted with a permanent mounting medium. For toluidine blue staining, the slides were stained with $0.1 \%$ toluidine blue stained for $2 \mathrm{~min}$, washed with tap water for $10 \mathrm{~min}$, and mounted with a permanent mounting medium. All staining procedures performed at RT. Stained slides were imaged by a Nikon eclipse Ti-U light microscope (Nikon Corporation).

Between February 2017 and May 2019, synovial fluid samples were collected from 34 patients with OA (eight men and 26 women; mean age, $53.7 \pm 16.1$ years) and 25 with RA (all women; mean age, 68.6 \pm 8.5 years) at Hanyang
University Hospital for Rheumatic Disease. For synovial fluid analysis, $10 \mathrm{ml}$ fluid was incubated with $1.5 \mathrm{mg}$ hyaluronidase (cat. no. H3506; Sigma-Aldrich) for $15 \mathrm{~min}$ at $37^{\circ} \mathrm{C}$, followed by centrifugation at $1,400 \mathrm{xg}$ for $15 \mathrm{~min}$ at $4^{\circ} \mathrm{C}$. After centrifugation, the fluids were immediately divided into aliquots and stored at $-80^{\circ} \mathrm{C}$ for $\mathrm{TNF} \alpha$ levels (cat. no. DTA00D; R\&D Systems, Inc.) measurement using ELISA according to manufacturer's protocol.

Isolation of human OA chondrocytes. Hyaline cartilage from OA knee joints was scraped using a rongeur and collected in serum-free DMEM (cat. no. L0103-500; Biowest) buffer containing $1 \mathrm{mg} / \mathrm{ml}$ Collagenase type 2 (cat. no. C6885; Sigma-Aldrich). The collected tissue samples were incubated at $37^{\circ} \mathrm{C}$ with agitation overnight. The next day, the digested hyaline cartilage was filtered through a $70-\mu \mathrm{m}$ strainer (cat. no. 93070; SPL Life Sciences) and seeded $\sim 60 \%$ cell density in DMEM (cat. no. L0103-500; Biowest) supplemented with 10\% FBS (cat. no. 16000-044; Gibco; Thermo Fisher Scientific, Inc.), $1 \%$ penicillin and streptomycin (cat. no. 15140122; Gibco; Thermo Fisher Scientific, Inc.) at $37^{\circ} \mathrm{C}$ and $5 \% \mathrm{CO}_{2}$. Primary chondrocytes at passage $2-5$ were used in subsequent experiments. Until the passage 2-5, chondrocytes were cultured at $60 \%$ density before full cell density at $37^{\circ} \mathrm{C}$.

Reagents and biological agents. Recombinant human TNF $\alpha$ (cat. no. 300-01A; PeproTech, Inc.), golimumab (Janssen Global Services, LLC; $1 \mu \mathrm{g} / \mathrm{ml}, 37^{\circ} \mathrm{C}, 4$ weeks) and BAY 11-7082 (cat. no. B5556; Sigma-Aldrich; 10 or $30 \mu \mathrm{M}, 37^{\circ} \mathrm{C}$, $24 \mathrm{~h})$ were obtained.

Flow cytometric analysis. The chondrocytes were fixed using a fixation/permeabilization solution kit (cat. no. 554715; BD Biosciences) and stained withCD34 (cat.no.343607; BioLegend, Inc.; 1:100),CD44(cat.no.338807;BioLegend,Inc.; 1:100),CD59 (cat.no. 304711; BioLegend, Inc.; 1:100), CD74 (cat. no. 326811; BioLegend, Inc.; 1:100), CD90 (cat. no. 328109; BioLegend, Inc.; 1:100), CD105 (cat. no. 323205; BioLegend, Inc.; 1:100), CD146 (cat. no. 361015; BioLegend, Inc.; 1:100), CD164 (cat. no. 324805; BioLegend, Inc.; 1:100), SOX9-Alexa-647 (cat. no. 565493; BD Pharmingen; BD Biosciences; 1:200), ACAN-PE (cat. no. sc-33695; Santa Cruz Biotechnology, Inc.; 1:100), IgG1-Alexa-647 (cat. no. 557732; BD Pharmingen; BD Biosciences; 1:100), IgG1-PE (cat. no. 400112; BioLegend, Inc.; 1:100), IgG1-APC (cat. no. 400122; BioLegend, Inc.; 1:100), IgG1-FITC (cat. no. 400109; BioLegend, Inc.; 1:100) or IgG2a-APC (cat. no. 400221; BioLegend, Inc.; 1:100) for $30 \mathrm{~min}$ at $4^{\circ} \mathrm{C}$. The dilution of the antibody for FACS was ranged from 1:100-1:200. After staining, cells were washed with Perm/Wash Buffer (cat. no. 554715; BD Biosciences) and analyzed by flow cytometry (FACS Canto II; BD Biosciences). Data were analyzed using FlowJo version 10.7 software (FlowJo LLC).

Proliferation assay. The water-soluble tetrazolium salt (WST) assay was performed with EZ-CYTOX (cat. no. EZ-1000; Dogen Bio Co., Ltd.) according to the manufacturer's instructions. Primary chondrocytes were plated into 96-well plate $\left(1 \times 10^{3}\right.$ cells/well) and treated with 10 or $25 \mathrm{ng} / \mathrm{ml} \mathrm{TNFa}$ 
in $37^{\circ} \mathrm{C} \mathrm{CO}_{2}$ incubator for 1-6 days. WST solution was added to the cells, which were incubated for $1 \mathrm{~h}$. The absorbance at $450 \mathrm{~nm}$ was measured with a microplate reader (Thermo Fisher Scientific, Inc.).

Human MMP antibody array. Chondrocytes were stimulated with $10 \mathrm{ng} / \mathrm{ml}$ human TNF $\alpha$ at RT for 1 day and collected for human MMP analysis. The stimulated cells were lysed in 1X RIPA buffer including phosphatase and proteinase inhibitors and assessed according to the manufacturer's protocol (cat. no. ab134004; Abcam). To analyze the array data, comparison of signaling intensities for individual spots was detected using the UVItech system (Cleaver Scientific Ltd.) and analyzed with ImageJ $1.52 \mathrm{a}$ version software (National Institutes of Health).

Reverse transcription-quantitative $(R T-q) P C R$. RT-qPCR was performed as previously described (20). Briefly, total RNA of the TNF-treated cells extracted with TRIzol ${ }^{\circledR}$ reagent (cat. no. 15596018; Invitrogen; Thermo Fisher Scientific, Inc.) was used to generate complementary DNA $\left(42^{\circ} \mathrm{C}\right.$ for $1 \mathrm{~h}$ and then $70^{\circ} \mathrm{C}$ for $10 \mathrm{~min}$ ) using a RevertAid First Strand cDNA Synthesis kit (cat. no. K1622; Thermo Fisher Scientific, Inc.). qPCR was performed on a CFX96 Real-time PCR detection system (cat. no. 1855201; Bio-Rad Laboratories, Inc.) following the manufacturer's procedure and the following thermal cycling: Initial denaturation for $3 \mathrm{~min}$ at $95^{\circ} \mathrm{C}$; two step-cycling: Denaturation for $10 \mathrm{sec}$ at $95^{\circ} \mathrm{C}$, combined annealing/extension for $30 \mathrm{sec}$ at $60^{\circ} \mathrm{C}$ for 35 cycle. The expression of each target gene was normalized to GAPDH. Normalized expression values were averaged and the relative levels of gene expression were quantified by using the comparative CT method (21). Primers used for PCR were as follows: MMP1 forward, 5'-AGAGCAGATGTGGACCATGC-3' and reverse, 5'-TTGTCCCGATGATCTCCCCT-3'; MMP3 forward, 5'-TCTATGGACCTCCCCCTGAC-3' and reverse, 5'-GATTTGCGCCAAAAGTGCCT-3'; MMP13 forward, 5'-GCCATTACCAGTCTCCGAGG-3' and reverse, 5'-TAC GGTTGGGAAGTTCTGGC-3'; SOX9 forward, 5'-CTGAAC GAGAGCGAGAAGCG-3' and reverse, 5'-CCCGTTCTTCAC CGACTTCC-3'; ACAN forward, 5'-TGGGAACCAGCCTAT ACCCCAG-3' and reverse, 5'-CAGTTGCAGAAGGGCCTT CTGTAC-3'; COL2 forward, 5'-GCCGGATCTGTGTCTGTG AC-3' and reverse, 5'-TGTCCCTTTGGTCCTGGTTG-3'; and GAPDH forward, 5'-CAAGATCATCAGCAATGCC-3' and reverse, 5'-CTGTGGTCATGAGTCCTTCC-3'.

Immunoblotting. Immunoblot analysis was performed as previously described (22). The treated cells were lysed in RIPA buffer (50 mM Tris- $\mathrm{HCl}$ (pH 8.0), $150 \mathrm{mM} \mathrm{NaCl}, 0.1 \%$ SDS, $0.6 \%$ Na-deoxycholate, $1 \%$ Triton X-100) supplemented with protease (cat. no. 535140, Calbiochem) and phosphatase (cat. no.5870, Cell signaling) inhibitor cocktails. Lysed samples were incubated on ice for $1 \mathrm{~h}$ followed by a centrifugation at $12,000 \mathrm{x}$ g for $30 \mathrm{~min}$ at $4^{\circ} \mathrm{C}$. The protein of whole lysates were determined using a Bradford protein assay (cat. no. 5000006; Bio-Rad Laboratories, Inc.). Protein (30 50 $\mu \mathrm{g})$ were separated by SDS-PAGE and electrophoretically transferred onto nitrocellulose membranes (cat. no. 10600002, Cytiva) in a transbuffer. Membranes were blocked with 5\% non-fat milk in
Tris-buffered saline (TBS) with $0.1 \%$ Tween-20 and incubated with specific primary antibodies, followed by incubation with horseradish peroxidase-conjugated secondary antibodies. The dilution of the primary antibody for Immunoblotting was ranged from 1:500 to 1:1,000. Diluted primary antibodies were incubated at $4^{\circ} \mathrm{C}$ overnight and secondary antibodies incubation diluted 1:1,000 at RT for $1 \mathrm{~h}$. Membranes were visualized with Pierce ECL (cat. no. 34580; Thermo Fisher Scientific, Inc.) and the visualized images were collected by chemiluminescence imaging system (Alliance Q9 advanced, Uvitech System). The primary antibodies for TNF receptor 1 (cat. no. sc-8436; 1:1,000) and NF- $\mathrm{B}$ p65 (cat. no. sc-372; 1:1,000) were from Santa Cruz Biotechnology, Inc. Phosphorylated (p-)NF- $\kappa$ B p65 (cat. no. 3033; 1:1,000), p-ERK (cat. no. 9101s; 1:1,000), total-ERK (cat.no. 9102s; 1:1,000), p-p38 (cat.no.9215s; 1:500), total-p38 (cat. no. 9212; 1:1,000), and $\beta$-actin (cat. no. 4970; 1:5,000) antibodies were from Cell Signaling Technology, Inc. MMP-1 (cat. no. MAB901; 1:500), MMP-3 (cat. no. MAB513; 1:500), and MMP-13 (cat. no. MAB511; 1:500) antibodies were from R\&D Systems, Inc. Goat anti-rabbit (cat. no. 111-035-003; $1: 2,000)$ and anti-mouse (cat. no. 115-035-003; 1:2,000) secondary antibodies were from Jackson ImmunoResearch Laboratories, Inc.

Trichloroacetic acid (TCA) assay. TCA precipitation was performed as previously described (23). In brief, chondrocytes were seeded at $80-90 \%$ confluence. The next day, growth medium was replaced with serum-free DMEM including $10 \mathrm{ng} / \mathrm{ml} \mathrm{TNF} \alpha$ or distilled water for 1 day. The cell supernatant obtained by centrifugation at $1,224 \mathrm{x} \mathrm{g}$ at $4^{\circ} \mathrm{C}$ for $10 \mathrm{~min}$ was collected for TCA precipitation, subjected to $12.5 \%$ SDS-PAGE and then stained with Coomassie Brilliant Blue R-250 solution (cat. no. C2006; Biosesang) as a loading control. After destaining (acetic acid: Methanol: Water; 7.5:5:87.5) overnight, the gel was washed five times with distilled water for $10 \mathrm{~min}$ and followed by immunoblotting procedures.

Immunofluorescence. The TNF $\alpha$-stimulated chondrocytes were washed twice with 1X PBS and fixed with $10 \%$ formalin at RT for $15 \mathrm{~min}$, followed by permeabilization with $1 \mathrm{X}$ PBS containing $0.1 \%$ Triton X-100 and 1\% BSA (cat. no. BSA-BSH-1XG; Rocky Mountain Biologicals, Inc.) at $\mathrm{RT}$ for $1 \mathrm{~h}$, incubation with a primary antibody at $4^{\circ} \mathrm{C}$ overnight, washing with $1 \mathrm{X}$ PBS and incubation with Cy3-conjugated anti-rabbit antibody (cat. no. 111-165-144; Jackson Immunoresearch) or Alexa 488-conjugated anti-mouse antibody (cat. no. A-11001; Invitrogen) for $1 \mathrm{~h}$. All primary and secondary antibodies were used at 1:100 dilution. The stained cells were washed with distilled water and mounted with DAPI (cat. no. H1200; Vector Laboratories, Inc.; Maravai Life Sciences). In order to visualize stained cells, immunofluorescence images were collected with a confocal microscope (TCS SP5; Leica Microsystems GmbH). Images were captured using LAS version 4.2.1 software (Leica Microsystems GmbH).

Promoter assay. NF-кB p65 wild-type and two p65 S536A (substitution of alanine for serine) or S536E (substitution of glutamate for serine 536) mutant promoters in pGL3-Basic were a gift from Dr Heekyoung Chung (Hanyang University, 
A

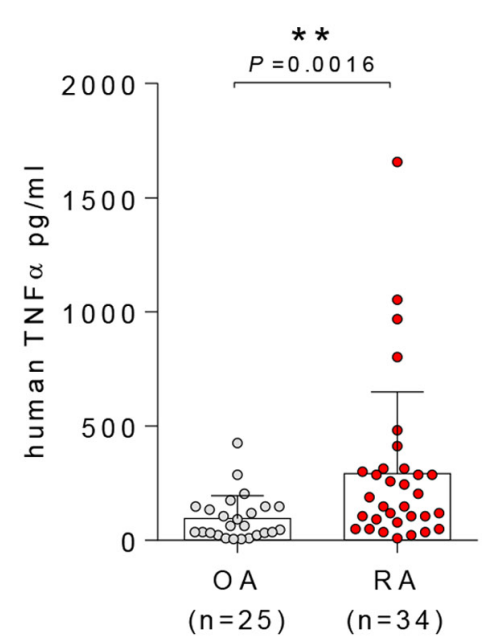

B
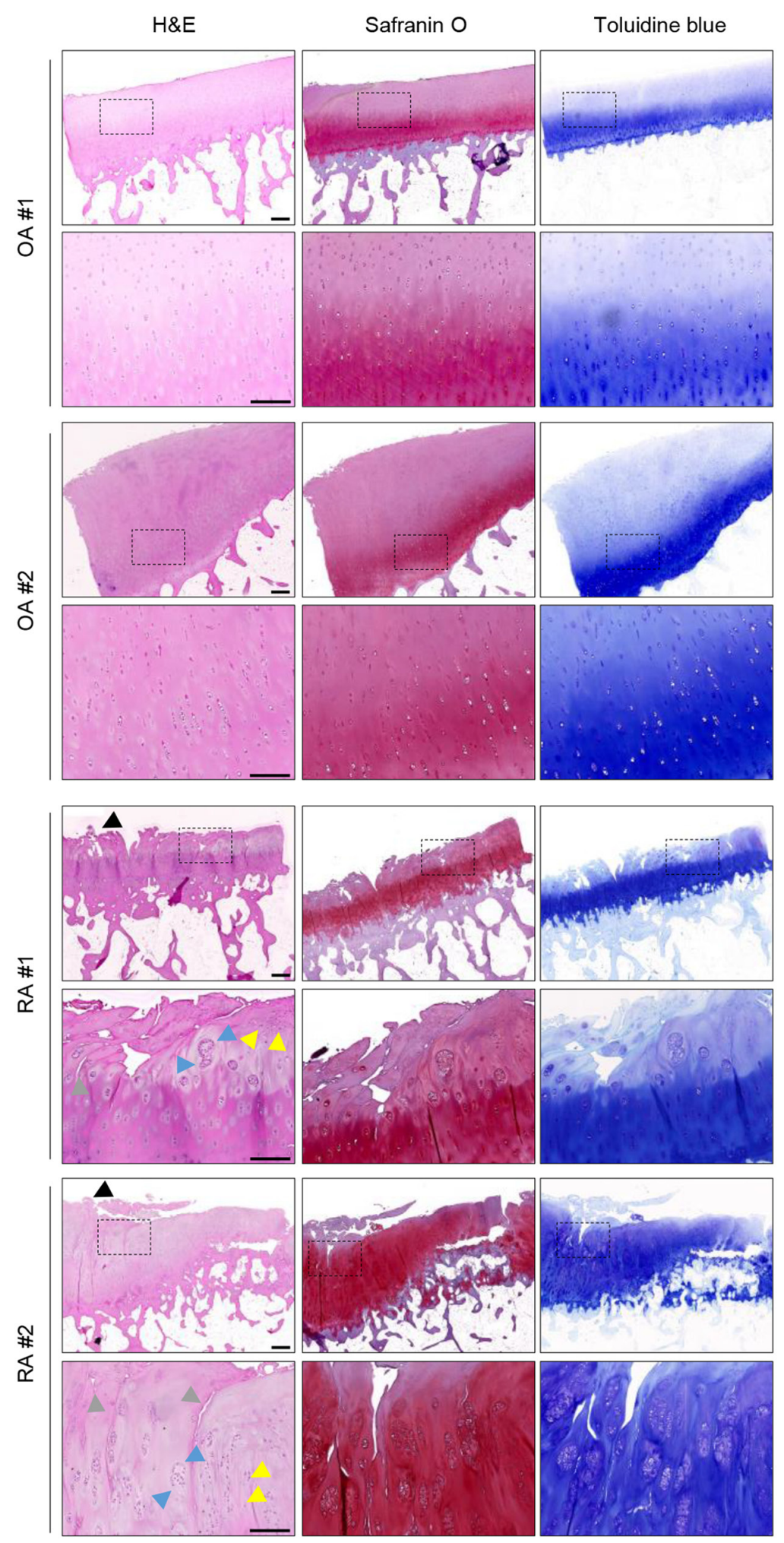

Figure 1. High TNF $\alpha$ levels in synovial fluid and destruction of hyaline cartilage are observed in RA. (A) TNF $\alpha$ levels in OA ( $\mathrm{n}=25)$ and RA ( $\mathrm{n}=34)$ synovial fluid were assessed by ELISA. ${ }^{* *} \mathrm{P}<0.01$, unpaired Student's t-test. (B) Knee tissue samples (including hyaline cartilage) from patients with OA and RA were stained with H\&E, Safranin O and Toluidine Blue. Overt fibrillation indicated by black arrows; Chondyocyte clustering indicated by blue arrows; changes in chondrocyte morphology/distribution indicated by yellow arrows; matrix destruction indicated by grey arrows. Scale bar, $200 \mu \mathrm{m}$. RA, rheumatoid arthritis; $\mathrm{OA}$, osteoarthritis; $\mathrm{H} \& \mathrm{E}$, hematoxylin and eosin; TNF $\alpha$, tumor necrosis factor $\alpha$.

Seoul, South Korea) (24). 293T cells were a generous gift from Dr Heekyoung Chung (Hanyang University, Seoul, Republic of Korea) and seeded into $60-\mathrm{mm}$ culture plates $\left(2 \times 10^{5}\right.$ cells per well) and co-transfected with p65 wild-type, p65 S536A or p65 S536E (1 $\mu \mathrm{g} / \mathrm{well})$, and Renilla $(0.25 \mu \mathrm{g} / \mathrm{well})$ plasmids as control for $48 \mathrm{~h}$ using Lipofectamine ${ }^{\circledR} 3000$ 
A
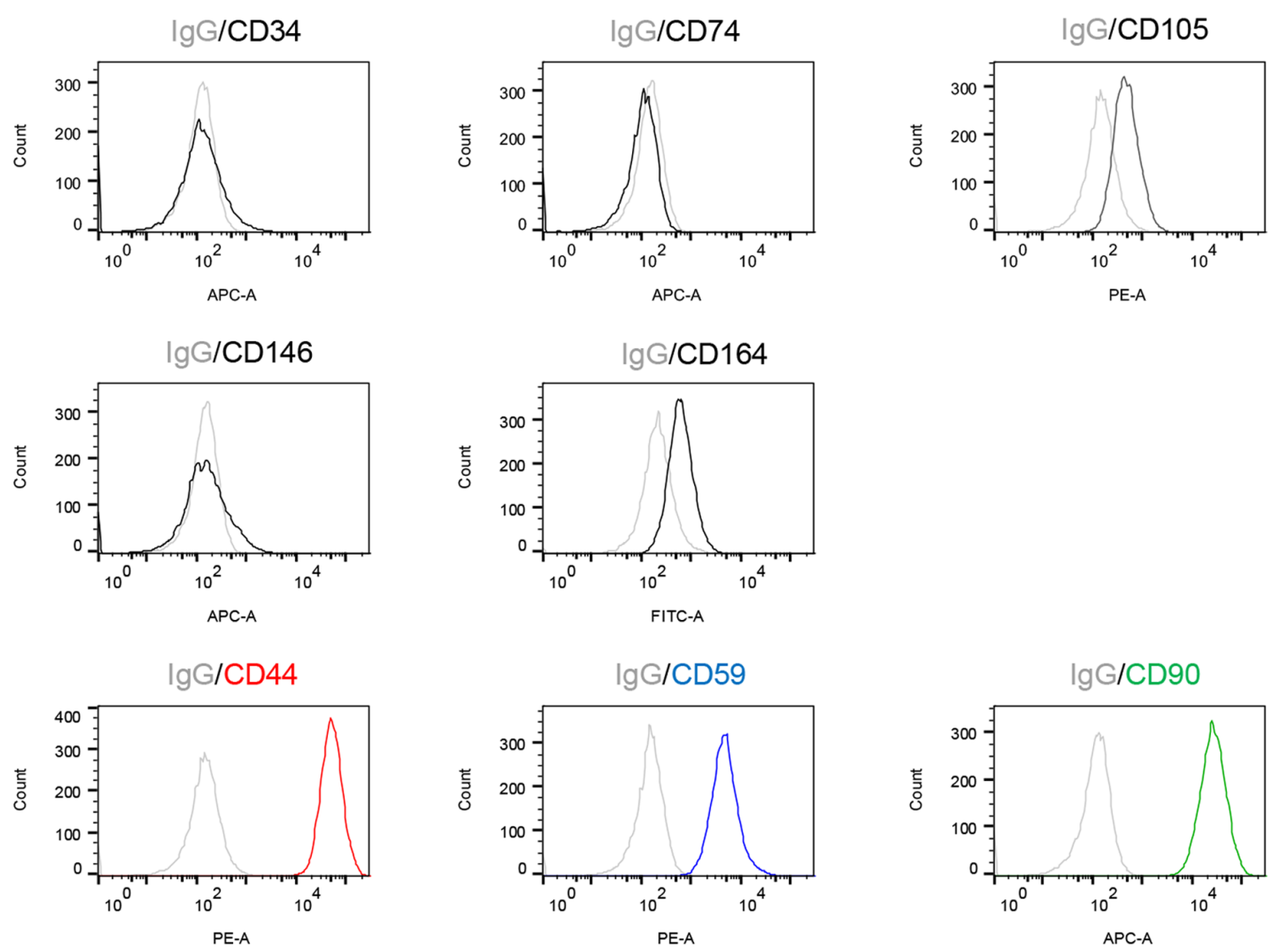

B
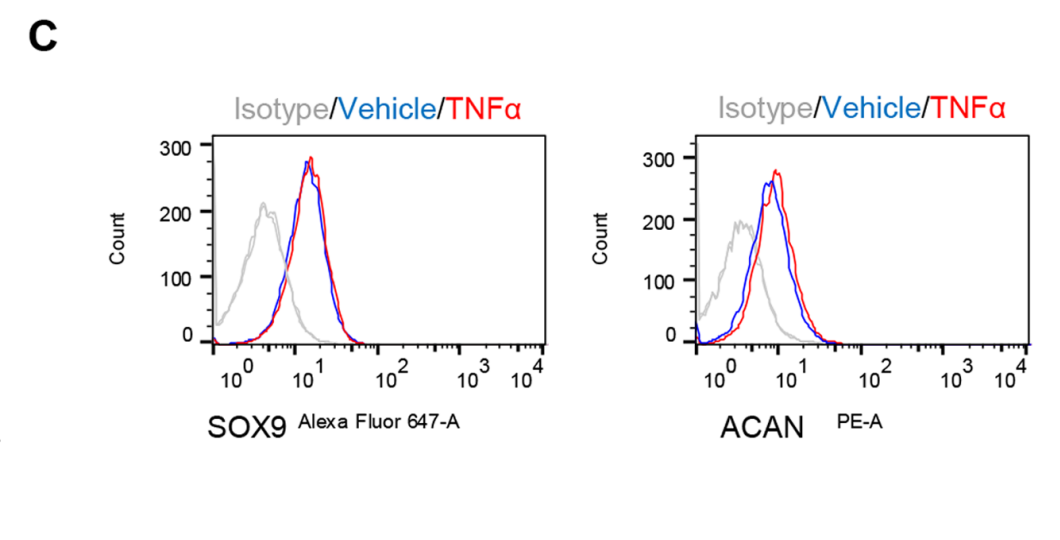

Figure 2. TNF $\alpha$ does not significantly affect proliferation and extracellular molecule expression in OA chondrocytes. (A) CD34, CD44, CD59, CD74, CD90, CD105, CD146 and CD164 surface markers of chondrocytes were evaluated by flow cytometry. (B) Chondrocytes were exposed to TNF $\alpha$ and assessed by water-soluble tetrazolium salt assay. $\mathrm{n}=5$, one-way ANOVA with Tukey's post hoc test. (C) Chondrocytes were exposed to $25 \mathrm{ng} / \mathrm{ml} \mathrm{TNF} \alpha$ for $24 \mathrm{~h}$ and assessed by flow cytometry. IgG was used as a control. OA, osteoarthritis; TNF $\alpha$, tumor necrosis factor $\alpha$; ACAN, aggrecan; SOX9, SRY-box transcription factor 9.

(cat. no. L3000-015; Invitrogen; Thermo Fisher Scientific, Inc.). The transfected cells were reseeded on a 12 -well culture plate $\left(5 \times 10^{4}\right.$ cells per well) for treatment at $37^{\circ} \mathrm{C}$ for $24 \mathrm{~h}$ with distilled water or 10 or $25 \mathrm{ng} / \mathrm{ml} \mathrm{TNF} \alpha$ and then analyzed with Dual-Luciferase Reporter Assay system (cat. no. E1500; Promega Corporation). The procedure was performed according to the manufacturer's instructions and activity was measured with a luminometer (Titertek-Berthold). The measured values were analyzed by comparison with Renilla luciferase. This ratio was then normalized to the averaged ratio of vehicle.
Chondrogenic differentiation of chondrocytes with pellet culture. The 'pellet culture' method was performed as previously described (25). Briefly, primary chondrocytes $\left(2 \times 10^{5}\right)$ were collected following centrifugation at $25^{\circ} \mathrm{C}$ for $3 \mathrm{~min}$ at $441 \mathrm{x} \mathrm{g}$, replaced and cultured in chondrogenic medium with distilled water or TNF $\alpha$ for 4 weeks. The medium was changed every 3 days. Chondrogenic medium was composed of serum-free DMEM/F12 (cat. no. 11320033; Gibco; Thermo Fisher Scientific, Inc.) with $10 \%$ Insulin-Transferrin-Selenium premix tissue culture supplement (cat. no.I3146; Sigma-Aldrich; Merck KGaA), $10 \mu \mathrm{M}$ dexamethasone (cat. no. D-2915; 
A

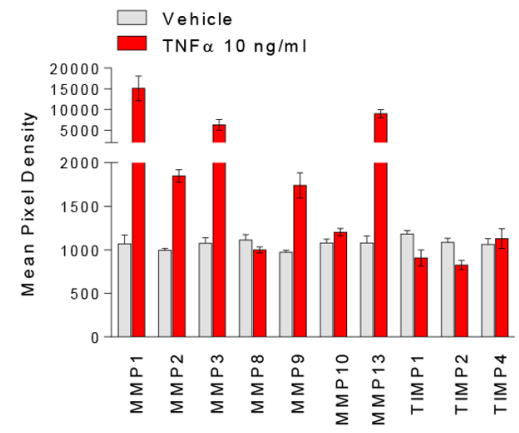

D



Stimulated-Cells Sup.
B

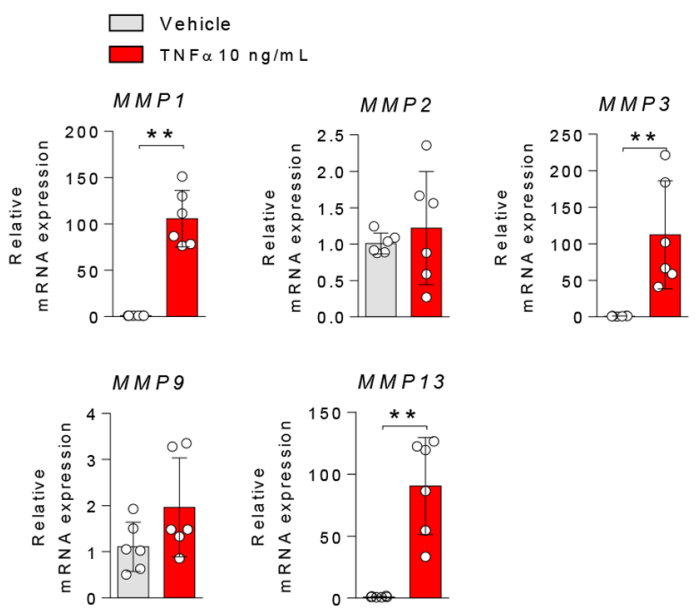

E
C

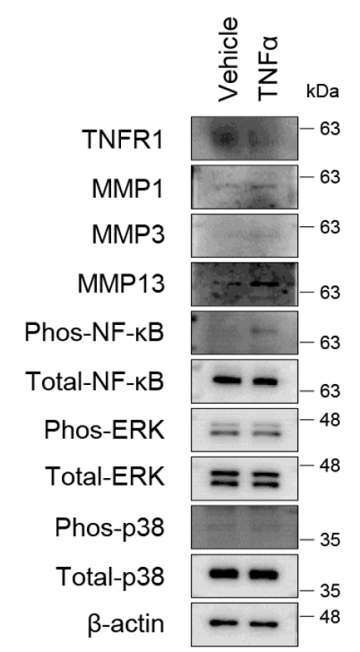

$\mathbf{F}$


Scale bar: $50 \mu \mathrm{m}$

Figure 3. TNF $\alpha$ induces the expression of MMPs in OA chondrocytes via NF- $\mathrm{BB}$ activation. (A) Chondrocytes were treated with vehicle or $10 \mathrm{ng} / \mathrm{ml} \mathrm{TNF} \alpha$ for $24 \mathrm{~h}$. MMP protein expression levels in stimulated cell lysates were semi-quantified via a human MMP antibody array (n=2). (B) $M M P$ mRNA expression in stimulated cells was determined by reverse transcription-quantitative PCR. (C) TNF $\alpha$-treated chondrocytes were assessed by immunoblotting. Phosphorylation was semi-quantified with ImageJ and calculated relative to each total protein. (D) Proteins secreted in the cell supernatant by TNF $\alpha$ stimulation were precipitated with trichloroacetic acid and detected by immunoblotting. Coomassie blue staining was used as a loading control. (E) 293T cells were transfected with p65 wild type or double mutants (S536S or S536E), followed by treatment with vehicle, $10 \mathrm{or} 25 \mathrm{ng} / \mathrm{ml} \mathrm{TNF} \alpha$ for $24 \mathrm{~h}$; promoter activity analysis was

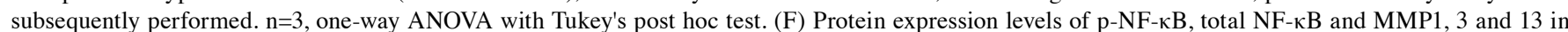
$\mathrm{TNF} \alpha$-stimulated cells were analyzed by immunofluorescence. Representative data are shown. Scale bar, $50 \mu \mathrm{m} .{ }^{*} \mathrm{P}<0.05,{ }^{* *} \mathrm{P}<0.01 ;{ }^{* * *} \mathrm{P}<0.001$ (mean $\pm \mathrm{SD}$; $\mathrm{n}=6$ ). OA, osteoarthritis; phos, phosphorylated; TNF $\alpha$, tumor necrosis factor $\alpha$; MMP, matrix metalloproteinase; NF- $\mathrm{kB}$, nuclear factor $\kappa \mathrm{B}$.

Sigma-Aldrich; Merck KGaA), $1 \mu \mathrm{M}$ ascorbate-2-phosphate (cat. no. 49752; Sigma-Aldrich; Merck KGaA), $1 \%$ sodium pyruvate (cat. no. 11360070; Gibco; Thermo Fisher Scientific,
Inc.) and $10 \mathrm{ng} / \mathrm{ml}$ TGF- $\beta 1$ (cat. no. 0218209-1; PeproTech, Inc.). $10 \%$ Formalin-fixed pellets at RT for $24 \mathrm{~h}$ were washed with 1X PBS and transferred to $30 \%$ sucrose solution overnight. 
A

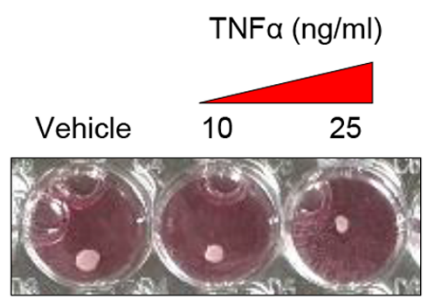

B

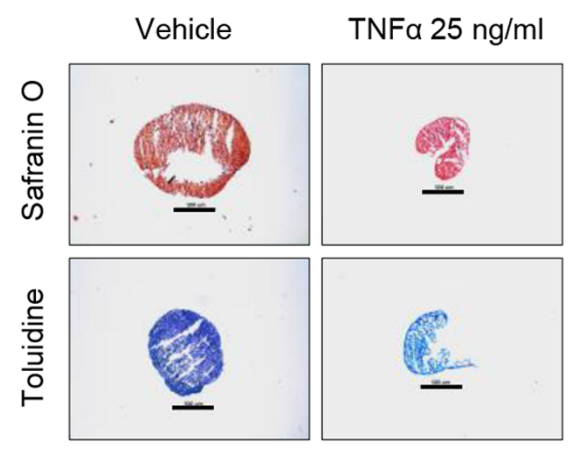

C

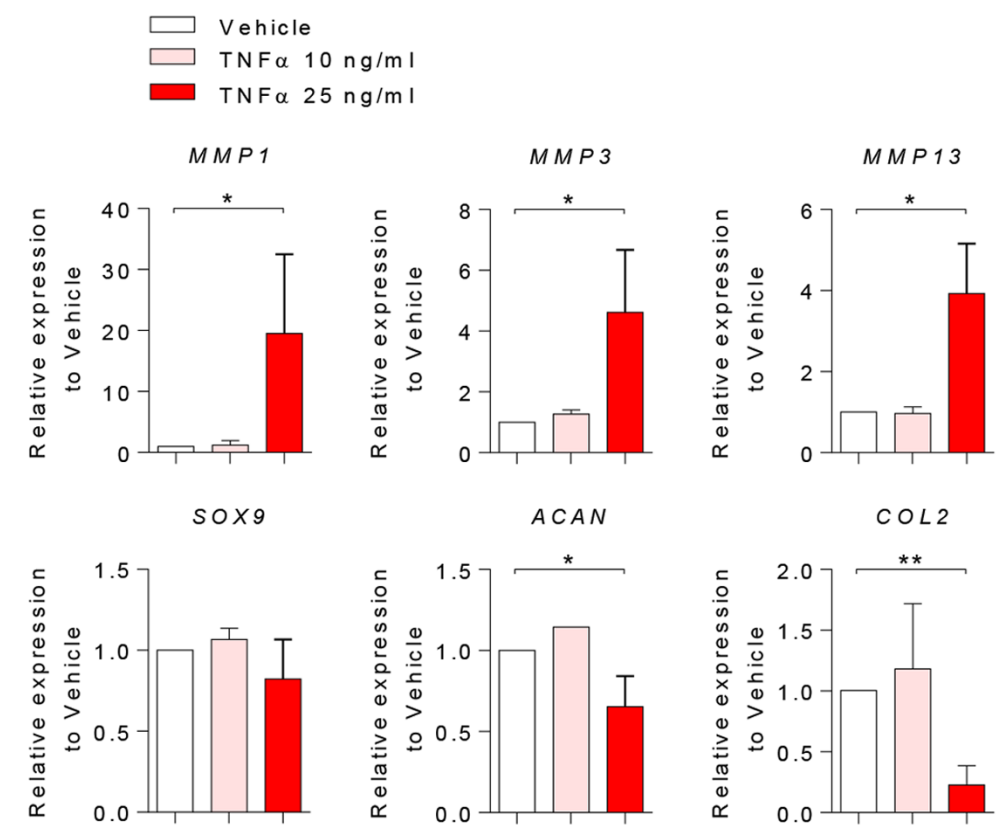

Figure 4. TNF $\alpha$ inhibits chondrogenic differentiation of osteoarthritis chondrocytes. Chondrocytes were induced with chondrogenic differentiation media for 28 days in the presence of vehicle, or 10 or $25 \mathrm{ng} / \mathrm{ml} \mathrm{TNF} \alpha$. (A) Images were captured after 28 days differentiation. Differentiating chondrocytes were (B) stained by Safranin O and Toluidine Blue and (C) analyzed by reverse transcription-quantitative PCR. Scale bar, $500 \mu \mathrm{m}$. Data are presented as the mean \pm SD $(n=4) .{ }^{*} \mathrm{P}<0.05 ;{ }^{* *} \mathrm{P}<0.01$. COL2, collagen type 2; ACAN, aggrecan; MMP, matrix metalloproteinase; SOX9, SRY-box transcription factor 9; $\mathrm{TNF} \alpha$, tumor necrosis factor $\alpha$.

Pellets were soaked in OCT compound (cat. no. 4583; Sakura Finetek USA) and sectioned on a cryotome (cat. no. CM1850; Leica Microsystems $\mathrm{GmbH}$ ) to create $15-\mu \mathrm{m}$ sections on gelatin-coated slides. Then, the slides were stained with Safranin O (cat. no. 1446640250; ACROS Organics) and Toluidine blue (cat. no. T3260-5g; Sigma-Aldrich; Merck $\mathrm{KGaA}$ ). Each staining was washed with water for $1 \mathrm{~min}$ and then stained with $0.1 \%$ dyeing solution for $1 \mathrm{~min}$ at RT. Stained slides were imaged under a Nikon eclipse Ti-U light microscope (Nikon Corporation).

Statistical analysis. Data were analyzed with GraphPad Prism 6 software (GraphPad Software, Inc.). A two-tailed Student t-test was used to compare data between two unpaired groups. One-way ANOVA with Tukey's post hoc test was used to compare data between more than two groups. All data are expressed as the mean $\pm \mathrm{SD}(\mathrm{n} \geq 3)$. $\mathrm{P}<0.05$ was considered to indicate a statistically significant difference.

\section{Results}

High TNFa levels in synovial fluid and destruction of hyaline cartilage are observed in RA. TNF $\alpha$ levels in synovial fluid were significantly higher in patients with RA than OA (mean, 168.7 vs. 64.28 pg/ml; Fig. 1A). Although superficial fibrillation and loss of proteoglycan detection with safranin $\mathrm{O}$ staining were observed in OA hyaline cartilage, patients with RA exhibited more severe hyaline cartilage damage than patients with OA. Overt fibrillation (black arrows), chondrocyte clustering (blue arrows), changes in chondrocyte morphology/distribution (yellow arrows) and matrix destruction (grey arrows) were observed in patients with RA (Fig. 1B).

TNF $\alpha$ has no significant effects on proliferation and extracellular molecule expression in OA chondrocytes. The present study aimed to detect known chondrocyte surface markers and investigate the effects of TNF $\alpha$ on them $(22,23)$. CD44, CD59 and CD90 were highly expressed in chondrocytes, whereas CD34, CD74 and CD146 were not; in addition, there was partial positive expression of CD105 and CD164. (Fig. 2A). Following TNF $\alpha$ treatment, there were no significant changes in the rate of cell proliferation (Fig. 2B) or chondrocyte expression of SOX9 and ACAN (Fig. 2C).

TNF $\alpha$ induces MMP expression in human OA chondrocytes. TNF $\alpha$-treated chondrocytes were used as a model for inflammatory arthritis. In order to determine whether TNF $\alpha$ may affect the destructive hyaline cartilage of patients with inflammatory arthritis, chondrocytes were stimulated with TNF $\alpha$. To confirm dose effects of TNF $\alpha$ in chondrocytes, we treated with 1, 5, 10, 25, $50 \mathrm{ng} / \mathrm{ml}$ TNF $\alpha$ dose and analyzed MMP expression levels using RT-qPCR and immunoblotting. We confirmed an increase in MMP1, 3, 13 expression in dose-dependently TNF $\alpha$ treatment (Fig. S1). Strong increases in the protein expression levels of MMP1, 3 and 13, and a decrease in TIMP metallopeptidase inhibitor (TIMP)1 and TIMP2 expression levels were observed (Figs. 3A, S2 and S3). Changes in the expression levels of MMPs were validated by RT-qPCR (Fig. 3B) and immunoblotting (Fig. 3C). TNF $\alpha$ stimulation of chondrocytes elevated MMP1, 3, and 13 protein expression levels in the cytoplasm and led to extracellular secretion of these proteins (Fig. 3C, D and F). 


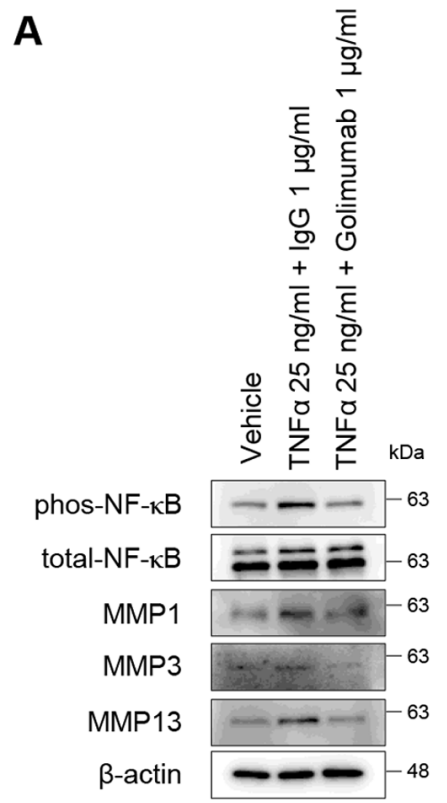

C

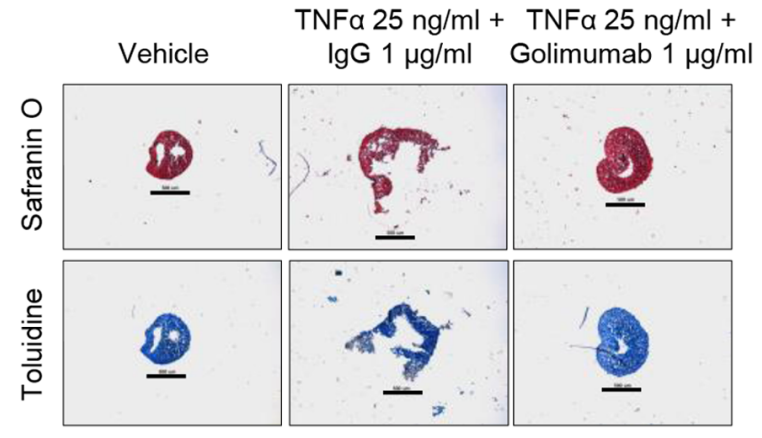

B

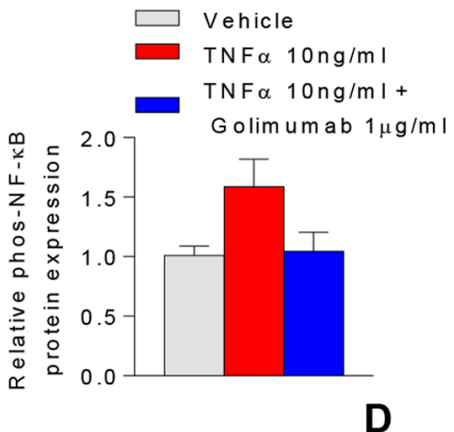

$N F-\kappa B$ element



TNF $\alpha(\mathrm{ng} / \mathrm{m} \mathrm{I})$

$\lg G(1 \mu \mathrm{g})$

Golimumab $(1 \mu \mathrm{g})$

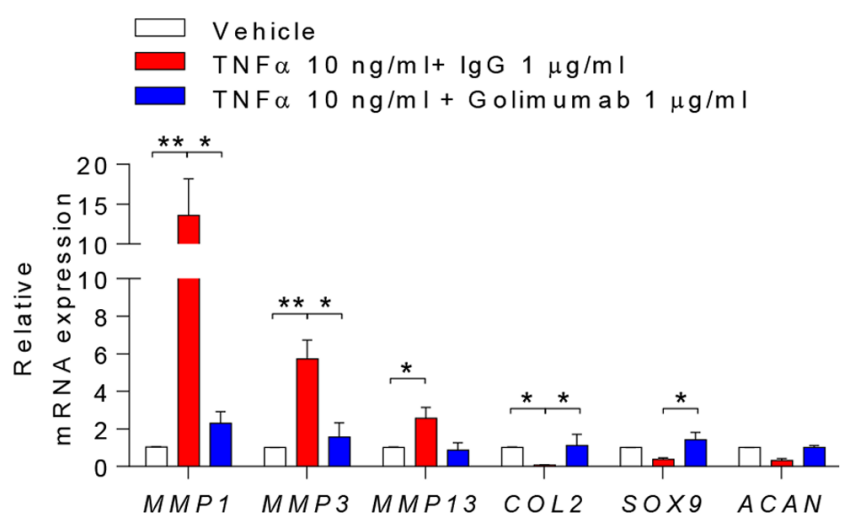

Figure 5. Blocking TNF $\alpha$ attenuates TNF $\alpha$-driven degradation of the extracellular matrix of osteoarthritis chondrocytes. (A) Chondrocytes were treated for $24 \mathrm{~h}$ and analyzed by immunoblotting. Golimumab was used as an anti-TNF agent ( $\mathrm{n}=3$ ). P-NF- $\mathrm{KB}$ protein was semi-quantified with ImageJ and calculated

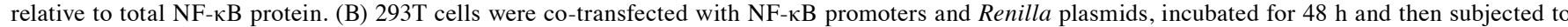
luciferase activity assay. (C) Chondrocytes were stimulated with TNF $\alpha$ or a TNF $\alpha$ blocker during chondrogenic differentiation for 28 days and then analyzed by Safranin $\mathrm{O}$ and Toluidine Blue staining. (D) mRNA expression was analyzed by reverse transcription-quantitative $\mathrm{PCR} . \mathrm{n}=3,{ }^{*} \mathrm{P}<0.05 ;{ }^{* *} \mathrm{P}<0.01 ;{ }^{* * *} \mathrm{P}<0.001$, one-way ANOVA with Tukey's post hoc test. phos, phosphorylated; COL2, collagen type 2; ACAN, aggrecan; MMP, matrix metalloproteinase; SOX9, SRY-box transcription factor 9; TNF $\alpha$, tumor necrosis factor $\alpha$.

Moreover, TNF $\alpha$ induced NF- $\mathrm{B}$ phosphorylation and nuclear translocation in chondrocytes, and significantly augmented p65 promoter activity, but not that of mutants (Fig. 3E and F). Therefore, it was concluded that TNF $\alpha$ promoted the expression of MMP1, 3 and 13 in human chondrocytes, which was accompanied by activation of $\mathrm{NF}-\kappa \mathrm{B}$ signaling.

TNF $\alpha$ decreases the regenerative capacity of $O A$ chondrocytes during differentiation. In order to elucidate the effect of TNF $\alpha$ on regenerative capacity in chondrocytes, chondrogenic differentiation of chondrocytes was induced in the presence or absence of TNF $\alpha$ treatment. Chondrocytes treated with TNF $\alpha$ exhibited lower differentiation capacity; they showed weaker intensity of staining and smaller pellet size compared with controls (Fig. 4A and B). Furthermore, upregulation of $M M P 1, M M P 3$ and $M M P 13$, and downregulation of $A C A N$ and COL2 was observed (Fig. 4C), which suggested that TNF $\alpha$ stimulation induced a metabolic shift from anabolism to catabolism during chondrogenic differentiation. Thus, it was concluded that TNF $\alpha$ decreased the regenerative capacity of chondrocytes during differentiation.

Blocking TNF $\alpha$ attenuates TNF $\alpha$-driven cartilage matrix degradation in $O A$ chondrocytes. To determine whether the modulation of TNF $\alpha$ affected catabolic and anabolic gene expression levels, human chondrocytes were treated with $1 \mu \mathrm{g} / \mathrm{ml}$ golimumab (a TNF $\alpha$ blocker) in the presence of TNF $\alpha$. TNF $\alpha$ stimulation upregulated $\mathrm{p}-\mathrm{NF}-\kappa \mathrm{B}, \mathrm{MMP} 1, \mathrm{MMP} 3$ and MMP13 in chondrocytes, whereas treatment with golimumab impeded these changes mediated by TNF $\alpha$ (Fig. 5A). In the line with Fig. 5A, changes in TNF-mediated $\mathrm{p}-\mathrm{NF}-\kappa \mathrm{B}$ were quantified (Fig. 5A, right panel). TNF $\alpha$ activated the WT NF- $\kappa$ B promoter in a dose-dependent manner but blocking $\mathrm{TNF} \alpha$ diminished this activation (Fig. 5B). In addition, treatment with 10 or $30 \mu \mathrm{M}$ $\mathrm{BAY}$, an NF- $\mathrm{B}$ inhibitor, suppressed TNF $\alpha$-mediated induction of $M M P 1, M M P 3$ and $M M P 13$ mRNA expression in 
chondrocytes (Fig. S4). During chondrogenic differentiation, treatment with the TNF $\alpha$ blocker interfered with the destructive effect of TNF $\alpha$ on physical changes of chondrogenesis (Fig. 5C) and its mRNA expressions (Fig. 5D). Therefore, blocking TNFa may attenuate $\mathrm{TNF} \alpha$-driven cartilage matrix degradation in inflammatory arthritis.

\section{Discussion}

The present study revealed that $\mathrm{TNF} \alpha$ levels were increased in synovial fluid and the destruction of hyaline cartilage was greater in RA compared with in OA. To demonstrate the association between $\mathrm{TNF} \alpha$ and hyaline cartilage destruction in $\mathrm{OA}$, chondrocytes were stimulated with TNF $\alpha$; the results indicated that TNF $\alpha$ induced a metabolic shift in chondrocytes via NF- $\mathrm{kB}$ signaling. Based on these findings, a model for the pathogenesis of hyaline cartilage degradation in inflammatory arthritis was proposed. TNF $\alpha$, an inflammatory cytokine that is increased by excessive inflammation, may modulate anabolic and catabolic factors, thereby revealing potential therapeutic targets for inflammatory arthritis progression.

The precise mechanism by which $\mathrm{TNF} \alpha$ suppresses the expression of matrix proteoglycans, such as ACAN and COL2, requires further study. It was hypothesized that MMP 1, 3 and 13 are indicators of TNF $\alpha$-driven matrix proteoglycan degradation. Although 24-h TNFa exposure did not affect SOX9 and ACAN expression in chondrocytes, exposure to TNF during chondrogenic differentiation resulted in decreased mRNA expression levels of anabolic mediators, such as $A C A N$ and $C O L 2$. Exposure of chondrocytes to TNF $\alpha$ did not affect cell proliferation, but high dose-exposure may induce cell death and senescence (26,27). Moreover, loss of matrix proteoglycans was revealed to be $\mathrm{TNF} \alpha$-dependent because TNF $\alpha$ blockade inhibited destruction of chondrocytes by TNF $\alpha$. Therefore, anti-TNF $\alpha$ therapy may alleviate the destruction of matrix proteoglycans and hyaline cartilage in inflammatory arthritis.

When chondrocytes become hypertrophic, they express unique genes, such as $C O L 10$ and $M M P 13$; these genes indicate calcification or matrix mineralization $(28,29)$. In line with these results, the present study showed that TNF $\alpha$ treatment upregulated COL10 mRNA expression (data not shown). Collectively, these data suggested that TNF $\alpha$ stimulation of chondrocytes regulated MMP13 and COL10 expression, leading to chondrocyte hypertrophy, calcification or matrix mineralization.

$\mathrm{TNF} \alpha$ plays a critical role in bone destruction in several types of inflammatory joint disease $(30,31)$. Particularly in $\mathrm{RA}$, focal bone loss occurs due to excessive bone resorption by osteoclasts. Moreover, bone formation by osteoblasts is impaired at the bone erosion site. Thus, anti-TNF therapies could delay the progression of bone destruction without bone erosion in patients with RA, leading to significant clinical improvement. TNF $\alpha$ in inflammatory arthritis has a destructive function in all of the components that comprise joints, namely osteoclasts, osteoblasts, and chondrocytes (32-34).

Blocking studies with an anti-TNF $\alpha$ agent in an arthritis model have shown that TNF $\alpha$ may drive cartilage and bone destruction $(35,36)$. Treatment with TNF $\alpha$ inhibitors decreased inflammation, as well as bone and cartilage damage, in patients with RA $(30,31)$. Although osteoclastogenesis is a more dominant mechanism in the bone erosion and cartilage destruction of inflammatory arthritis $(8,34)$, there are fewer reports on the pathological mechanisms by which TNF $\alpha$ and its inhibitors influence physiological changes of hyaline cartilage in human knee joints.

$\mathrm{OA}$ is a degenerative joint disease caused by the degradation of hyaline cartilage. Cartilage loss in OA is associated with aging and mechanical stress, as well as inflammation. The primary cause of OA is aging and mechanical stress, which suppress the regenerative capacity of chondrocytes involved in extracellular matrix components comprising hyaline cartilage. Moreover, total knee replacement for OA is a significant health burden, and there are no therapeutic drugs available yet for the regeneration of hyaline cartilage $(37,38)$. Thus, there is a significant unmet need for effective medical therapies for $\mathrm{OA}$. The present study demonstrated that TNF $\alpha$ contributed to OA pathogenesis via NF- $\mathrm{KB}$ mechanisms affecting metabolic imbalance in chondrocytes, leading to structural joint damage (39). Similarly, it was recently demonstrated that patients with OA exhibited a notable response to anti-TNF therapy (40), indicating a pivotal role for TNF $\alpha$ in cartilage catabolism. Thus, TNF inhibitors may be a therapeutic option for patients with $\mathrm{OA}$ with severe inflammation or $\mathrm{TNF} \alpha$-driven cartilage destruction.

In conclusion, the present study showed that TNF $\alpha$ was associated with progressive destruction of hyaline cartilage in OA. Mechanistically, chondrocyte exposure to TNFa increased $M M P 1, M M P 3$ and $M M P 13$ gene expression, causing degradation of ACAN and COL2 structural proteins in hyaline cartilage. During chondrogenic differentiation, TNF $\alpha$ stimulation decreased the expression of $C O L 2$ and $A C A N$, but not $S O X 9$, thereby decreasing relative chondrogenic size; these effects were reversed following treatment with anti-TNF agents. Therefore, treatment with anti-TNF therapy may relieve chondrocyte destruction in inflammatory arthritis.

\section{Acknowledgements}

The authors would like to thank Dr Dae Hyun Yoo, Dr Sang-Cheol Bae and Dr Jae-Bum Jun of the Hanyang University Hospital for Rheumatic Disease for helping to collect synovial fluid from patients. Immunofluorescence images were analyzed by confocal microscopy (Leica Microsystems $\mathrm{GmbH}$ ) at Hanyang LINC Analytical Equipment Center (Seoul, Korea).

\section{Funding}

The present study was supported by the Basic Science Research Program through the National Research Foundation of Korea (NRF) and funded by the Ministry of Science, ICT, and Future (grant nos. NRF-2016R1A2B4008606 and 2019R1A2C2004214). It was also supported by a Korea Health Technology R\&D grant through the Korea Health Industry Development Institute, which is funded by the Ministry of Health and Welfare, Republic of Korea (grant no. HI17C0888).

\section{Availability of data and materials}

The datasets used and/or analyzed during the current study are available from the corresponding author on reasonable request. 


\section{Authors' contributions}

JP, HP, YLL and SW performed all experiments. SJ, BN, JHY, and YGK designed the experiments. JP, HP, and SJ analyzed experimental data. JHY provided human knee joint samples. YGK and BN provided synovial fluid of patients with OA and RA. SJ and THK wrote the manuscript. JP, HP, and SJ confirm the authenticity of all the raw data. THK conceptualized and supervised the study. All authors read and approved the final manuscript.

\section{Ethics approval and consent to participate}

Studies involving human materials were performed in compliance with the Helsinki Declaration and were approved by the Ethics Committee of Hanyang University Hospital (approval no. IRB-2017-05-003) and Hanyang University Guri Hospital (approval no. 2018-07-024). All subjects provided written informed consent.

\section{Patient consent for publication}

Not applicable.

\section{Competing interests}

The authors declare that they have no competing interests.

\section{References}

1. Goldring MB: Articular cartilage degradation in osteoarthritis. HSS J 8: 7-9, 2012.

2. Goldring MB and Marcu KB: Cartilage homeostasis in health and rheumatic diseases. Arthritis Res Ther 11: 224, 2009.

3. Langer R and Vacanti JP: Tissue engineering. Science 260: 920-926, 1993

4. Mao AS and Mooney DJ: Regenerative medicine: Current therapies and future directions. Proc Natl Acad Sci USA 112: 14452-14459, 2015.

5. Jiang S, Guo W, Tian G, Luo X, Peng L, Liu S, Sui X, Guo Q and Li X: Clinical application status of articular cartilage regeneration techniques: Tissue-engineered cartilage brings new hope. Stem Cells Int 2020: 5690252, 2020.

6. Malda J, Groll J and van Weeren PR: Rethinking articular cartilage regeneration based on a 250 -year-old statement. Nat Rev Rheumatol 15: 571-572, 2019.

7. Mueller MB and Tuan RS: Anabolic/Catabolic balance in pathogenesis of osteoarthritis: Identifying molecular targets. PM R 3 (Suppl 1): S3-S11, 2011.

8. Little CB, Flannery CR, Hughes CE, Mort JS, Roughley PJ, Dent C and Caterson B: Aggrecanase versus matrix metalloproteinases in the catabolism of the interglobular domain of aggrecan in vitro. Biochem J 344: 61-68, 1999.

9. Matyas JR, Ehlers PF, Huang D and Adams ME: The early molecular natural history of experimental osteoarthritis I. Progressive discoordinate expression of aggrecan and type II procollagen messenger RNA in the articular cartilage of adult animals. Arthritis Rheum 42: 993-1002, 1999.

10. Park EH, Kim JS, Lee JS, Lee YJ, Song YW and Lee EY Compound $\mathrm{K}$ inhibits interleukin-1 $\beta$-induced expression of inflammatory mediators and matrix metalloproteinases by inhibiting mitogen-activated protein kinase activation in chondrocytes. J Rheumatic Dis 25: 188, 2018.

11. Billinghurst RC, Dahlberg L, Ionescu M, Reiner A, Bourne R, Rorabeck C, Mitchell P, Hambor J, Diekmann O, Tschesche H, et al: Enhanced cleavage of type II collagen by collagenases in osteoarthritic articular cartilage. J Clin Invest 99: 1534-1545, 1997.

12. Shlopov BV, Gumanovskaya ML and Hasty KA: Autocrine regulation of collagenase 3 (matrix metalloproteinase 13) during osteoarthritis. Arthritis Rheum 43: 195-205, 2000.
13. Tetlow LC, Adlam DJ and Woolley DE: Matrix metalloproteinase and proinflammatory cytokine production by chondrocytes of human osteoarthritic cartilage: Associations with degenerative changes. Arthritis Rheum 44: 585-594, 2001.

14. Aigner T, Fundel K, Saas J, Gebhard PM, Haag J, Weiss T, Zien A, Obermayr F, Zimmer R and Bartnik E: Large-scale gene expression profiling reveals major pathogenetic pathways of cartilage degeneration in osteoarthritis. Arthritis Rheum 54: 3533-3544, 2006.

15. Tracey KJ and Cerami A: Tumor necrosis factor: A pleiotropic cytokine and therapeutic target. Annu Rev Med 45: 491-503, 1994.

16. Liu G: Molecular mechanism and TNF signaling and beyond. Cell Res 15: 24-27, 2005

17. Kammermann JR, Kincaid SA, Rumph PF, Baird DK and Visco DM: Tumor necrosis factor-alpha (TNF-alpha) in canine osteoarthritis: Immunolocalization of TNF-alpha, stromelysin and TNF receptors in canine osteoarthritic cartilage. Osteoarthritis Cartilage 4: 23-34, 1996.

18. Arend WP and Dayer JM: Inhibition of the production and effects of interleukin-1 and tumor necrosis factor alpha in rheumatoid arthritis. Arthritis Rheum 38: 151-160, 1995.

19. Kapoor M, Martel-Pelletier J, Lajeunesse D, Pelletier JP and Fahmi H: Role of proinflammatory cytokines in the pathophysiology of osteoarthritis. Nat Rev Rheumatol 7: 33-42, 2011.

20. Jo S, Yoon S, Lee SY, Kim SY, Park H, Han J, Choi SH, Han JS, Yang $\mathrm{JH}$ and Kim TH: DKK1 induced by 1,25D3 is required for the mineralization of osteoblasts. Cells 9: 236, 2020.

21. Livak KJ and Schmittgen TD: Analysis of relative gene expression data using real-time quantitative PCR and the 2(-Delta Delta C(T)) method. Methods 25: 402-408, 2001.

22. Jo S, Wang SE, Lee YL, Kang S, Lee B, Han J, Sung IH, Park YS, Bae SC and Kim TH: IL-17A induces osteoblast differentiation by activating JAK2/STAT3 in ankylosing spondylitis. Arthritis Res Ther 20: 115, 2018.

23. Koontz L: TCA precipitation. Methods Enzymol 541: 3-10, 2014.

24. Kim H, Chung H, Kim HJ, Lee JY, Oh MY, Kim Y and Kong G: Id-1 regulates $\mathrm{Bcl}-2$ and $\mathrm{Bax}$ expression through $\mathrm{p} 53$ and NF-kappaB in MCF-7 breast cancer cells. Breast Cancer Res Treat 112: 287-296, 2008.

25. Lee JK, Jo S, Lee YL, Park H, Song JS, Sung IH and Kim TH: Anterior cruciate ligament remnant cells have different potentials for cell differentiation based on their location. Sci Rep 10: 3097, 2020

26. Li X, Du W, Ma FX, Feng X, Bayard F and Han ZC: High concentrations of TNF- $\alpha$ induce cell death during interactions between human umbilical cord mesenchymal stem cells and peripheral blood mononuclear cells. PLoS One 10: e0128647, 2015.

27. Li P, Gan Y, Xu Y, Song L, Wang L, Ouyang B, Zhang C and Zhou Q: The inflammatory cytokine TNF- $\alpha$ promotes the premature senescence of rat nucleus pulposus cells via the PI3K/Akt signaling pathway. Sci Rep 7: 42938, 2017.

28. Kishimoto H, Akagi M, Zushi S, Teramura T, Onodera Y, Sawamura T and Hamanishi C: Induction of hypertrophic chondrocyte-like phenotypes by oxidized LDL in cultured bovine articular chondrocytes through increase in oxidative stress. Osteoarthritis Cartilage 18: 1284-1290, 2010.

29. Borzi RM, Olivotto E, Pagani S, Vitellozzi R, Neri S, Battistelli M, Falcieri E, Facchini A, Flamigni F, Penzo M, et al: Matrix metalloproteinase 13 loss associated with impaired extracellular matrix remodeling disrupts chondrocyte differentiation by concerted effects on multiple regulatory factors. Arthritis Rheum 62: 2370-2381, 2010.

30. Jung SM, Kim KW, Yang $\mathrm{CW}$, Park $\mathrm{SH}$ and $\mathrm{Ju} \mathrm{JH}$ : Cytokine-mediated bone destruction in rheumatoid arthritis. J Immunol Res 2014: 263625, 2014.

31. Karmakar S, Kay J and Gravallese EM: Bone damage in rheumatoid arthritis: Mechanistic insights and approaches to prevention. Rheum Dis Clin North Am 36: 385-404, 2010.

32. Azuma Y, Kaji K, Katogi R, Takeshita S and Kudo A: Tumor necrosis factor-alpha induces differentiation of and bone resorption by osteoclasts. J Biol Chem 275: 4858-4864, 2000. 
33. Gilbert L, He X, Farmer P, Boden S, Kozlowski M, Rubin J and Nanes MS: Inhibition of osteoblast differentiation by tumor necrosis factor-alpha. Endocrinology 141: 3956-3964, 2000.

34. Lofvall H, Newbould H, Karsdal MA, Dziegiel MH, Richter J, Henriksen K and Thudium CS: Osteoclasts degrade bone and cartilage knee joint compartments through different resorption processes. Arthritis Res Ther 20: 67, 2018.

35. van Schouwenburg PA, Rispens $\mathrm{T}$ and Wolbink GJ: Immunogenicity of anti-TNF biologic therapies for rheumatoid arthritis. Nat Rev Rheumatol 9: 164-172, 2013.

36. Scott DL and Kingsley GH: Tumor necrosis factor inhibitors for rheumatoid arthritis. N Engl J Med 355: 704-712, 2006.

37. Bedair H, Cha TD and Hansen VJ: Economic benefit to society at large of total knee arthroplasty in younger patients: A Markov analysis. J Bone Joint Surg Am 96: 119-126, 2014.
38. Global Burden of Disease Study 2013 Collaborators: Global, regional, and national incidence, prevalence, and years lived with disability for 301 acute and chronic diseases and injuries in 188 countries, 1990-2013: A systematic analysis for the Global Burden of Disease Study 2013. Lancet 386: 743-800, 2015.

39. Chow YY and Chin KY: The role of inflammation in the pathogenesis of osteoarthritis. Mediators Inflamm 2020: 8293921, 2020.

40. Chisari E, Yaghmour KM and Khan WS: The effects of TNF-alpha inhibition on cartilage: A systematic review of preclinical studies. Osteoarthritis Cartilage 28: 708-718, 2020.

This work is licensed under a Creative Commons Attribution-NonCommercial-NoDerivatives 4.0 International (CC BY-NC-ND 4.0) License. 